\title{
A User Driven Dynamic Circuit Network Implementation
}

\author{
Chin P. Guok, David W. Robertson, Evangelos Chaniotakis, Mary R. Thompson, William Johnston, Brian Tierney
}

Abstract_-The requirements for network predictability are becoming increasingly critical to the DOE science community where resources are widely distributed and collaborations are world-wide. To accommodate these emerging requirements, the Energy Sciences Network has established a Science Data Network to provide user driven guaranteed bandwidth allocations. In this paper we outline the design, implementation, and secure coordinated use of such a network, as well as some lessons learned.

Index Terms - Computer network management, Network servers, Dynamic virtual circuits, Traffic control

\section{INTRODUCTION}

$\mathrm{T}$ HE ubiquity of best-effort IP routing in the internet today has proven to be both one of its greatest strengths and weaknesses. Best-effort routing is opportunistic, simplistic, and resilient. However it lacks predictability and flexibility, and does not provide any guarantees. These shortcomings have increasingly become the focus of the U.S Department of Energy (DOE) science community [1]. Today's sciences are increasingly driven by highly-distributed large-scale multidisciplinary collaborations which rely on high-speed networks to connect resources to process and integrate data from observations and simulations. The reliance on such networks is becoming a critical factor to the success of these collaborations.

An example of this is the USLHCNet [2] which was designed and implemented primarily to support the multinational collaborative Large Hadron Collider (LHC) [3] project. The LHC is located on the French-Swiss border near Geneva, Switzerland, with computational and storage resources distributed throughout the world. The LHC project is expected to produce roughly $40 \mathrm{~TB}$ [4] of data per day and

This work was supported by the Director, Office of Science. Office of Advanced Scientific Computing Research. Mathematical, Information, and Computational Sciences Division under U.S. Department of Energy Contract No. DE-AC02-05CH11231. This is LBNL report number LBNL-60373.

C. Guok is with the Energy Sciences Network, Berkeley, CA 94720, USA (phone: 510-495-2279; fax: 510-486-6712; e-mail: chin@ es.net).

D. Robertson is with the Lawrence Berkeley National Laboratory, Berkeley, CA 94720, USA (e-mail: dwrobertson@ @lbl.gov).

E. Chaniotakis is with the Energy Sciences Network, Berkeley, CA 94720, USA (e-mail: haniotak@ es.net).

M. Thompson is with the Lawrence Berkeley National Laboratory, Berkeley, CA 94720, USA (e-mail: mrthompson@1bl.gov).

W. Johnston is with the Energy Sciences Network, Berkeley, CA 94720 , USA (e-mail: wej@es.net).

B. Tierney is with the Lawrence Berkeley National Laboratory, Berkeley, CA 94720, USA (e-mail: bltierney@lbl.gov). uses a hierarchical data storage and management architecture to disseminate the data world wide for collaborative analysis. Due to the immense volume of data that will be generated continually, and the distributed analysis system that is tailored to analyze the data as it is generated, predictable network capacity is crucial in preventing data from backing up at the higher tier sites and stalling the workflow pipeline.

This paper outlines the design and operation of a network that can support predictable bandwidth circuits, and a software system capable of guaranteeing bandwidth to multiple users in a secure manner. As network paths often traverse multiple network domains, ongoing collaboration and standardization efforts in the scientific network community are also described.

\section{II.ENERGY SCIENCES NETWORK}

\section{A. Network Architecture}

To address the growing need for guaranteed bandwidth by such large-scale collaborations as the LHC, the DOE Energy Science Network (ESnet) [5] has designed and implemented the Science Data Network (SDN), which is logically distinct from the IP core network. The ESnet IP core network is architected primarily to transport IP packets, while the ESnet SDN is engineered to support dynamic virtual circuits (VCs). The preference for best-effort IP packets to utilize the IP core network over the SDN is done primarily by manipulating OSPF metrics. In the event that the IP core network is bifurcated, IP traffic can traverse the SDN. In essence, the SDN can backup the IP core network. However, based on operational requirements by the science community consuming most of the SDN bandwidth, only partial backup is supported.

The restriction of how much best-effort IP can traverse the SDN is implemented using Quality of Service (QoS) [6] mechanisms as described in the next section.

\section{B. Virtual Circuit Implementation}

Internally within ESnet, OSPF-TE [7], MPLS-TE [8], and RSVP-TE [9] are used to manage MPLS-LSPs on which the dynamic layer 2 and layer $3 \mathrm{VC}$ services are built. Additionally, LDP [10] is used to support layer $2 \mathrm{VCs}$ as defined in the EoMPLS [11] protocol.

The management of bandwidth usage and congestion control on each IP core and SDN link is accomplished by VC admission control and by QoS parameters. ESnet supports three user classes of service: expedited-forwarding, best-effort, and scavenger [12]. The expedited-forwarding class is used strictly by the dynamic circuit services, the best-effort class is 
used primarily for IP traffic, and the scavenger class is selected when there is a requirement or preference to use any available bandwidth without impacting other classes of traffic.

TABLE I

QOS QUEUe PERCENTAGES

\begin{tabular}{lcccc}
\hline & $\begin{array}{c}\text { Network- } \\
\text { Control }^{1}\end{array}$ & $\begin{array}{c}\text { Expedited- } \\
\text { Forwarding }\end{array}$ & $\begin{array}{c}\text { Best- } \\
\text { Effort }\end{array}$ & Scavenger \\
\hline IP Core & $5 \%$ & $20 \%$ & $74 \%$ & $1 \%$ \\
SDN & $5 \%$ & $74 \%$ & $20 \%$ & $1 \%$ \\
\hline
\end{tabular}

Each class of service is guaranteed the bandwidth percentages assigned to it as depicted in Table I, but can burst higher if there is unused bandwidth available. For the IP core links, which are primarily for IP traffic, up to $20 \%$ of the bandwidth can be used for dynamic circuit services. However in the SDN, which is used primarily for dynamic circuit services, only $20 \%$ of the link capacity can be used to back up IP traffic if the IP core partitions.

\section{ON-DEMAND SECURE CIRCUIT AND ADVANCED RESERVATION SYSTEM}

\section{A. User-Driven Virtual Circuits}

The ESnet On-demand Secure Circuit and Advanced Reservation System (OSCARS) [13] supports user driven advanced reservations of dynamic VCs at layer 2 (Ethernet VLANs), and layer 3 (IP). In this capacity, OSCARS is used as a domain controller for network resources within ESnet. OSCARS also functions as an Inter-Domain Controller (IDC) [29] which has the capability to communicate with other domain controllers (further discussed in section IV).

One of the core objectives of OSCARS is to provide easily used functionality that allows application programmers and end-users without a network engineering background to set up reservations for VCs. These can be requested on-demand, or scheduled in advance to support a workflow pipeline, for example to coincide with experimental data generation.

Two interfaces are provided: a SOAP-based messaging system for use by application programmers and for communication with similar systems used by other service providers (see the Collaborations section below), and a Web browser-based interface for the general user.

\section{B. Layer 2/3 VC Support}

For a user requested layer $2 \mathrm{VC}$, a VLAN identifier is negotiated at request time, and is subsequently used to determine packets designated for the layer $2 \mathrm{VC}$. This requires some coordination on the customer edge device to configure the correct VLAN tagging of the packets before entering the provider edge (PE) device.

For layer $3 \mathrm{VCs}$, the PE device filters on IP flow specifications (e.g. source/destination address/port, protocol,

\footnotetext{
1 Network-Control class-of-service is not user accessible but is included in the table for completeness
}

DSCP [14] bits) as submitted in the user VC request, to inject the selected packets into the layer $3 \mathrm{VC}$.

\section{Path Computation}

OSCARS utilizes a graph-based algorithm to determine the path for a circuit reservation request. Topology and capacity information is harvested from the network devices once an hour and is then imported into the OSCARS topology database. When a new circuit reservation request is received, a base topology graph is generated from the database taking into account any existing reservations whose time ranges overlap with the new reservation.

Vertices in the graph represent network devices as well as physical and logical interfaces, while edges represent "contains/belongs-to" or "connects to" relationships. For ESnet's production network the base topology graph currently contains approximately 1000 vertices and 1500 edges. Each edge in the graph has a cost associated with it, which has been engineered such that path computation is heavily biased towards a minimum-latency path over the SDN links. However, IP core links can be utilized (up to 20\%) where SDN resources are not available. Edge costs range from zero, in the case of links internal to a network device, to low (latency-dependent) values for SDN links, to very high values for IP core links.

Path computation is subsequently performed on the base topology graph taking into account the parameters and constraints specified in the $\mathrm{VC}$ reservation, such as source and destination endpoints, bandwidth, or VLAN tagging. From the base graph, vertices and edges are removed that do not match the reservation constraints, given the reservations currently scheduled for the time range of the requested reservation. For example, physical interfaces are removed that do not have enough bandwidth capacity available at the time the new reservation is scheduled, or that cannot support the required VLAN tag. This trimming process both ensures that the calculated path will satisfy the constraints, and has the beneficial side-effect of significantly reducing the number of vertices and edges in the graph. Finally, a Dijkstra shortestpath algorithm [15] is run on the modified topology graph, and the resulting path used to provision the $\mathrm{VC}$. If the trimming process results in a partitioned graph, a path cannot be calculated between the source and destination. The circuit reservation will subsequently have to be resubmitted with different parameters.

In addition to the above, a select set of trusted users are allowed to provide path computation steering hints. Path steering is performed by allowing the user to provide an ordered list of intermediate hops in addition to the source and destination. A path segment is then calculated between each pair of hops as provided. These segments are stitched together to form the complete path. Another option for trusted users is to manually specify the entire path, bypassing the path computation process entirely, but subject to the constraints 
given above.

If path segments of the $\mathrm{VC}$ need to be handled by other service providers, the request is forwarded to that provider. After the complete end-to-end path is set up, the reservation parameters, including the start time (which may be immediate), end time (which may be up to 10 years in the future), and path description are stored in the resource scheduling database, and will be taken into account when another $\mathrm{VC}$ reservation is requested.

\section{Circuit Setup and Automated Device Configuration}

Once a reservation is made, a VC setup can be instantiated either by a signal from the user, or by a separate "alarm-clock" process that periodically queries the database for reservations whose start times have been reached. This "alarm-clock" process also enforces reservation end times for all reservations, although a user may signal a teardown before the end time.

There are several router platforms deployed within ESnet, primarily Juniper and Cisco devices. Device specific configuration templates are used to initiate RSVP signaling, and MPLS LSP setup and teardown on the network devices. Specified parts of the template are replaced by user variables and OSCARS-generated reservation parameters at VC setup time. In the case of layer $3 \mathrm{VCs}$, where reservations are unidirectional, the configuration instance is pushed only to the PE router closest to the source (as defined by the reservation request). For layer $2 \mathrm{VCs}$, which are implemented using EoMPLS and are therefore bi-directional, device specific configuration instances are pushed to both $\mathrm{PE}$ routers at the start and end of the intra-domain VC. Teardown of VCs is done using complementary templates.

Note that it can take several minutes to configure a network device. See the Future Work section on plans to mitigate this.

\section{E. Authentication and Authorization}

Since a limited resource (bandwidth) is being reserved and claimed, and network devices are being manipulated (i.e. configuration changes, signaling), $\mathrm{VC}$ creation and setup needs to be done in a secure and auditable fashion.

The basic user authentication and authorization process is described in an earlier paper [13]. To briefly summarize here: requests coming through the SOAP API are authenticated by message signatures using X.509 certificates and keys. (The DOE science community has a well developed PKI infrastructure to support this sort of authentication. See http://www.doegrids.org/.) Requests coming through the Web browser-based interface use a registered user name and password combination. Authorization policy is kept locally in database tables, and is roughly grouped by a small number of role attributes which are assigned to each user.

The roles and permissions have evolved as the OSCARS system has been used. Originally there were two roles: user, who can make an unlimited reservation, see, modify, and signal (set up or tear down the circuit) his own reservations; and engineer, who can make unlimited reservations, specify path elements, and see, modify, and signal all reservations. As the system moved into pre-production use, two more roles were added: site administrator, who can make unlimited reservations, and see, modify, and signal any reservation that begins or terminates at his site regardless of the request originator; and operator, who can see all reservations, but not make or modify any. The site administrator role was requested to allow a network engineer from any ESnet directly served customer site to have complete control over VCs into the site (i.e. terminating on the PE interface facing the site) in case of unexpected events, such as router failures or suspected abuses of the reservation system. The operator role was requested to allow a NOC operator to check if a failed network element was part of a production $\mathrm{VC}$ and notify the $\mathrm{VC}$ owner or the site administrator to reconfigure the $\mathrm{VC}$.

Support for authorization tokens has also been added to support the work done by collaborators at the University of Amsterdam [16]. A unique, hard-to-forge token is created per reservation by the final IDC in a multi-domain path, stored with the reservation at each IDC, and returned to the user. It can then be used to authorize the signaling of the reservation. The token fulfills two objectives: first, the time consuming authorization procedure only happens at create reservation time, while a quick check of the token authorizes actions at VC setup time; second, it allows one user to make a reservation and a different user to instantiate or tear down the VC. The second scenario would be useful when a long-term reservation (several months) is made for the use of an experiment, and the circuit set up and torn down numerous times by various experiment members when data actually needs to be transferred.

\section{F. Over-subscription and Soft Reservations}

The initial implementation of OSCARS strictly prevented bandwidth over-booking and over-subscription (explained in more detail below). The circumvention of over-booking was enforced at user-request time by the path computation and resource scheduling processes. Over-subscription was negated at usage time by hard policing each $\mathrm{VC}$ to the requested bandwidth.

Emerging requirements from customers using dynamic VCs have demonstrated a need for over-booking stemming from two general use cases: i. customer managed load-balancing, and ii. redundant backup. An example of the first case would be a customer requesting two 10Gbps VCs over a 10GE path. The concurrent use of both VCs cannot exceed the 10Gbps link speed. However, each VC can utilize the entire bandwidth if the other $\mathrm{VC}$ is idle. The objective of the customer is to have both VCs active, and manage the load-balancing of the VCs accordingly. In the second case, a customer wants two $\mathrm{VCs}$, a primary and a redundant backup. In some scenarios, both the primary and redundant paths may share common links. In both cases, the core issue is over-booking and not 
over-subscription, as each $\mathrm{VC}$ request is processed discretely. This results in the first $\mathrm{VC}$ request potentially reserving the entire bandwidth path, and the second $\mathrm{VC}$ request failing, even though usage of both VCs is closely related. A major disadvantage of supporting over-booking is the inability to guarantee bandwidth. An alternative solution is to prohibit over-booking, and intelligently handle over-subscribed traffic. To resolve this issue, OSCARS was modified to implement soft reservations.

Soft reservations in OSCARS support the notion of oversubscription. With every VC reservation, a bandwidth amount is submitted with the request. This bandwidth amount is used by the path computation process to determine if adequate network resources are available. The cumulative bandwidth requests are bounded by the physical capacity of the link and cannot be over-booked. However, the policing of $\mathrm{VC}$ reservations has been modified to remark over-subscribed packets instead of dropping them. In essence, a successful VC reservation will guarantee the requested bandwidth, but any traffic exceeding the threshold will compete for available capacity on the path. The mechanism used to implement this is QoS. Packets below the requested bandwidth threshold will have their MPLS EXP bits set to 4 and queued in the expedited-forwarding queue as it traverses the intra-domain MPLS LSP. Packets above the threshold are remarked for scavenger service (MPLS EXP set to 1) where they compete with other traffic in the scavenger queue for available bandwidth. The best-effort queue is not used for dynamic VC services in order to avoid competition with "regular" IP traffic, even on the SDN links, so as to ensure a backup strategy for the IP Core network.

\section{Collaborations}

DICE [17] is a consortium of research and education network service providers comprised of DANTE [18], Internet2 [19], USLHCnet/CalTech [2], and ESnet. There has been significant progress and ongoing work by the DICE Control Plane Working Group to develop a WSDL description for IDC communications. Inter-domain communications and intercontinental end-to-end $\mathrm{VC}$ management have been demonstrated successfully between the DICE participants in several venues, including SC07 [30], and GLIF WG Winter 08 [31].

In addition to interoperability, the Internet2 IDC and OSCARS share the same codebase. However the Internet2 Dynamic Circuit Network (DCN) [29] deploys different network elements from those of the ESnet SDN, and as a result, Internet2 uses the Dynamic Resource Allocation via GMPLS Optical Networks (DRAGON) [20] software for pathfinding and intra-domain VC signaling, and the IDC for scheduling and inter-domain communications.

TeraPaths [21] and LambdaStation [22] are two projects designed to dynamically manage site LAN resources. In addition to coordinating resource allocations between the site gateway and the end-host, they communicate with OSCARS and/or Internet2's IDC via clients using the WSDL description to create host-to-host VCs.

In an effort to standardize the inter-domain interface between other dynamic circuit services, there is an on-going involvement in the Open Grid Forum Network Measurements Working Group (OGF NMWG) [23] and Global Lambda Integrated Facility (GLIF) Control Plane and Grid Integration Middleware Working Group [24] communities. OSCARS uses the XML schemas defined by the OGF Network Monitoring Working Group (OGF-NMWG) in its API and follows these definitions closely in its internal topology and resource scheduling database. There are also close collaborations with other projects including AutoBAHN [25], Phosphorus [26], and g-Lambda [27] to design and develop the common resource scheduling API.

\section{NETWORK USE}

The OSCARS service currently deployed in ESnet is at the pre-production stage. The service instance running on the ESnet production network currently manages several longlived production-level VCs as well as provides the capability for short-lived circuits primarily for network research. At the time of writing, OSCARS manages 13 production VCs totaling 43 Gbps of guaranteed bandwidth, mostly used for LHCrelated traffic.

At present, the primary users of the ESnet OSCARS service are Fermi National Accelerator Laboratory and Brookhaven National Laboratory, both LHC tier 1 data center sites. In both cases, close co-operation with the site network coordinator is necessary to intelligently traffic engineer primary and secondary VCs. These customers have high bandwidth and high availability demands for their VCs, presenting a significant challenge to network engineering staff. The OSCARS software suite is also used to implement traffic engineering solutions involving bandwidth management, multiple VCs for resiliency, and rerouting VCs around faulty connections.

In addition to working closely with customer sites, the ESnet OSCARS instance peers with the Internet2 IDC to reserve and manage cross-domain Ethernet VLAN VCs. This functionality has been demonstrated formally at $\mathrm{SC} 07$, I2 FMM '07 [32], and I2 SMM '08 [33], and is also used informally based on day-to-day user-driven needs. It is worth noting here that OSCARS has recently been used to tear down and re-provision several production-level VCs that were affected by a major hardware upgrade. The necessary reprovisioning was completed with minimal downtime; all of the circuits were back in operation at most 60 minutes after the new hardware was operational.

In the short period that OSCARS has supported productionlevel VCs, its two production users have required a persistent level of service, and to self-manage the usage of the reserved VC bandwidth resources (see Section III. F.). This is contrary to the initial design goals of dynamic VCs, which assumed that bandwidth resources would only be reserved when needed, and 
returned when not in use. This model will be re-evaluated when the LHC starts producing real data, prompting periodic downloads of large chunks of physics data from T0 to T1, and $\mathrm{T} 1$ to $\mathrm{T} 2$ sites.

\section{FUTURE WORK}

\section{A. Outage Management}

Planning is underway for OSCARS to be able to communicate with ESnet's internal outage planning database. The aim is to be able to automatically reroute existing reservations around outages if at all possible, and return to the status quo after the outage with minimal downtime. This presents a significant challenge because all affected VC paths need to be recalculated on the topology graph that represents the network during the outage. New paths will be assigned based on a set of rerouting policies and strategies that will be formulated in cooperation with all the stakeholders including customers, operators, and network engineers.

\section{B. Multi-layer circuits}

Planning is also ongoing to provide an integrated multilayer circuit provisioning service that will be able to model and utilize the cross-layer adaptation points and capabilities in our network, and calculate a multi-layer path. For example, a layer 3 service may be provisioned over a layer 2 circuit, which in turn may be provisioned over a layer 1 lightpath.

\section{Optimizations}

The vast majority of VC requests currently come from middleware such as LambdaStation and TeraPaths. These usually generate requests for short-lived VCs that need to be set up as quickly as possible. This requirement was not part of the original design, which assumed a small number of longerlived VCs. This usage presents a challenge since VC scheduling and setup is a relatively slow process involving network device configuration that currently requires at least several seconds and up to a few minutes in the case of multidomain VCs.

Significant modifications to the code are required to parallelize these time-consuming operations as much as possible. A full-featured scheduler component will be implemented that will simultaneously connect to and configure all relevant network devices to set up or tear down a VC. To avoid concurrent device configuration operations overwriting each other, the scheduler component will have multiple configuration queues running in parallel, ensuring correctness and minimizing setup time.

\section{ACKNOWLEDGMENT}

The authors would like to thank Tom Lehman of the University of Southern California Information Sciences Institute East, John Vollbrecht and Andrew Lake of Internet2, and Afrodite Sevasti, Guy Roberts, and Radek Krzywania of GÉANT2 for their efforts in interoperability testing and ongoing collaboration.

\section{REFERENCES}

[1] High Performance Network Planning Workshop (2002) [Online]. Available: http://www.es.net/hypertext/requirements.html

[2] USLHCNet (2008) [Online]. Available: http://lhcnet.caltech.edu/

[3] Large Hadron Collider (2007) [Online]. Available: http://lhc.web.cern.ch/lhc/

[4] Ian Bird, "The LHC Computing Grid," presented at the EGEE, Mar. 18, 2008, p. 4 [Online]. Available: http://www.ts.infn.it/eventi/viaggioalcern/allegati/LCG-EGEE20080426.pdf

[5] ESnet (2008) [Online]. Available: http://www.es.net

[6] G.Huston, "RFC2990: Next Steps for the IP QoS Architecture," IETF RFC, Nov. 2000.

[7] D. Katz, K.Kompella, and D. Yeung, "RFC2370: Traffic Engineering (TE) Extensions to OSPF Version 2," IETF RFC, Sep. 2003.

[8] E. Rosen, A. Viswanathan, and R. Callon "RFC3031: Multiprotocol Label Switching Architecture," IETF RFC, Jan. 2001.

[9] D. Awduche, L. Berger, D. Gan, T. Li, V. Srinivasan, and G. Swallow, "RFC3209: RSVP-TE: Extensions to RSVP for LSP Tunnels," IETF RFC, Dec. 2001.

[10] L. Andersson, P. Doolan, N. Feldman, A. Fredette, and B. Thomas, "RFC3036: LDP Specification," IETF RFC, Jan. 2001.

[11] M. Lasserre and V. Kompella, "RFC:4762, Virtual Private LAN Service (VPLS) Using Label Distribution Protocol (LDP) Signaling," IETF RFC, Jan. 2007.

[12] S. Shalunov and B. Teitelbaum, "QBone Scavenger Service (QBSS) Definition," Internet2 Technical Report, Mar. 2001 [Online]. Available: http://qos.internet2.edu/wg/wg-documents/qbss-definition.txt

[13] C. Guok, D. Robertson, M. Thompson, J. Lee, B. Tierney, and W. Johnston, "Intra and Interdomain Circuit Provisioning Using the OSCARS Reservation System," IEEE Broadband Communications, Networks and Systems, BROADNETS 2006, Oct. 2006, pp. 1-8.

[14] K. Nichols, S. Blake, F. Baker, and D. Black, "RFC2474: Definition of the Differentiated Services Field (DS Field) in the IPv4 and IPv6 Headers," IETF RFC, Dec. 1998.

[15] E. Dijkstra, "A note on two problems in connexion with graphs," Numer. Math. 1 (1959), pp. 269-271.

[16] Leon Gommans, Franco Travostino, John Vollbrecht, Cees de Laat, and Robert Meijer, "Token-based authorization of Connection Oriented Network resources," GRIDNETS conference proceedings, Oct. 2004 [Online]. Available: http://www.broadnets.org/2004/workshoppapers/Gridnets/gommans.pdf

[17] DICE (2008) [Online]. Available: http://www.geant2.net/server/show/conWebDoc.1308

[18] DANTE (2008) [Online]. Available: http://www.dante.net

[19] Internet2 (2008) [Online]. Available: http://www.internet2.edu

[20] DRAGON (2008) [Online]. Available: https:// dragon.maxgigapop.net/

[21] S. Bradley, F. Burstein, L. Cottrell, B. Gibbard, D. Katramatos, Y. Li, S. McKee, R. Pope-scu, D. Stampf, and D. Yu, "TeraPaths: A QoSEnabled Collaborative Data Sharing Infra-structure for Peta-scale Computing Research," Computing in High Energy and Nuclear Physics (CHEP 2006), T.I.F.R., Mumbai, India, Feb. 13-17, 2006.

[22] A. Bobyshev, M. Crawford, et.al., "Lambda Station: On-Demand Flow Based Routing for Data Intensive Grid Applications Over Multitopology Networks," IEEE Broadband Communications, Networks and Systems, BROADNETS 2006, Oct. 2006, pp. 1-9.

[23] OGF NMWG (2008) [Online]. Available: http://nmwg.internet2.edu

[24] GLIF Control Plane and Grid Integration Middleware Working Group (2008) [Online]. Available: http://www.glif.is/workinggroups/controlplane/

[25] AutoBAHN (2008) [Online]. Available: http://www.geant2.net/server/show/nav.1952

[26] S.Figuerola, N.Ciulli, M.De Leenheer, Y.Demchenko, W.Ziegler, and A.Binczewski, "PHOSPHORUS: Single-step on-demand services across multi-domain networks for e-science," European Conference and Exhibition on Optical Communication'07, Sep. 16-20 2007. 
[27] g-Lambda (2008) [Online]. Available: http://www.g-lambda.net

[28] E. Chaniotakis, C. Guok, "IDC: Inter-Domain Controller API," presented at GLIF Control Plane WG, Hawaii, USA, Jan. 192008.

[29] Internet2 Dynamic Circuit Network (2008) [Online]. Available: http://www.internet2.edu/network/dc/

[30] SuperComputing '07, Nevada, USA, Nov. 10-16 2007, (2008) [Online:]. Available: http://sc07.supercomputing.org

[31] GLIF: Working Groups Winter 2008, Honolulu, HI, USA, Jan. 19-20 2008, (2008). [Online:] Available: http://www.glif.is/meetings/2008/winter/

[32] Internet2 Fall Member Meeting 2007, San Diego, CA, USA, Oct. 8-11 2007, (2008) [Online:] Available: http://events.internet2.edu/2007/fall$\underline{\mathrm{mm} /}$

[33] Internet2 Spring Member Meeting 2008, Arlington, VA, USA, Apr. 2123 2008, (2008) [Online:] Available): http://events.internet2.edu/2008/spring-mm/

[34] GÉANT2 (2008) [Online]. Available: www.geant2.net 